\title{
Compact stars and the evolution of binary systems
}

\author{
E. P. J. van den Heuvel \\ Astronomical Institute, “Anton Pannekoek”, University of Amsterdam, The Netherlands
}

Received 2011 January 31; accepted 2011 February 27

\begin{abstract}
The Chandrasekhar limit is of key importance for the evolution of white dwarfs in binary systems and for the formation of neutron stars and black holes in binaries. Mass transfer can drive a white dwarf in a binary over the Chandrasekhar limit, which may lead to a Type Ia supernova (in case of a $\mathrm{CO}$ white dwarf) or an AccretionInduced Collapse (AIC, in the case of an O-Ne-Mg white dwarf; and possibly also in some $\mathrm{CO}$ white dwarfs) which produces a neutron star. The direct formation of neutron stars or black holes out of degenerate stellar cores that exceed the Chandrasekhar limit, occurs in binaries with components that started out with masses $\geq 8 \mathrm{M}_{\odot}$.
\end{abstract}

This paper first discusses possible models for Type Ia supernovae, and then focusses on the formation of neutron stars in binary systems, by direct core collapse and by the AIC of O-Ne-Mg white dwarfs in binaries. Observational evidence is reviewed for the existence of two different direct neutron-star formation mechanisms in binaries: (i) by electron-capture collapse of the degenerate O-Ne-Mg core in stars with initial masses in the range of 8 to about $12 \mathrm{M}_{\odot}$, and (ii) by iron-core collapse in stars with inital masses above this range. Observations of neutron stars in binaries are consistent with a picture in which neutron stars produced by e-capture collapse have relatively low masses, $\sim 1.25 \mathrm{M}_{\odot}$, and received hardly any velocity kick at birth, whereas neutron stars produced by iron-core collapses are more massive and received large velocity kicks at birth. Many of the globular cluster neutron stars and also some of the neutron stars in low-mass binaries in the Galactic disk are likely to have been produced by AIC of O-Ne-Mg white dwarfs in binaries. AIC is expected to produce normal strongly magnetized neutron stars, which in binaries can evolve into millisecond pulsars through the usual recycling scenario.

Keywords : stars: binaries: general - stars: evolution - stars: white dwarfs - stars: neutron - stars: pulsars: general - stars: supernovae: general 


\section{Introduction}

The Chandrasekhar limit is of key importance for the evolution of white dwarfs in binary systems which receive mass from a companion star. This is now the favoured model for the origin of Type Ia supernovae, which are known to be excellent 'standard candles' (e.g. Riess et al. 1998; Phillips et al. 1999). Their use in cosmology has led to the discovery of dark energy (Schmidt et al. 1998; Perlmutter et al. 1999).

It was realized long ago (Hoyle \& Fowler 1960) that Carbon-Oxygen (CO) white dwarfs, which are the final products of stars less massive than about $8 \mathrm{M}_{\odot}$, still contain a large amount of nuclear fuel, and that the ignition of carbon under degenerate conditions in the interior of a white dwarf will lead to runaway nuclear fusion, converting most of the star into ${ }^{56} \mathrm{Ni}$, thereby causing the star to blow up in a gigantic explosion with energy equivalent to that of a supernova. Since hydrogen and helium are absent in the spectra of Type Ia supernovae, and these spectra are dominated by products expected from explosive carbon burning, these supernovae fit very well with the thermonucelar explosion model of a carbon-oxygen $(\mathrm{CO})$ white dwarf (Hoyle \& Fowler 1960; Nomoto 1982a).

In order to trigger carbon ignition the mass of the white dwarf has to grow to the Chandrasekhar limit. The only realistically conceivable way to achieve this, is by the transfer of matter from a companion star in a binary system. This led Whelan \& Iben (1973) to suggest that Type Ia supernovae originate from binaries in which a red giant star is transferring mass to a CO white dwarf. This is the so-called 'Single-Degenerate' (SD) model for Type Ia supernovae. This model was worked out for example by Nomoto (1982a,b).

Subsequently it was realized by Webbink (1984) and Iben \& Tutukov (1984) that wide binaries of intermediate mass (components between $\sim 2$ and $8 \mathrm{M}_{\odot}$ ) may after several stages of mass exchange and common-envelope evolution leave very close binary systems consisting of two CO white dwarfs. When their orbital periods are shorter than about one day these systems will within a Hubble time merge, due to loss of orbital angular momentum by emission of gravitational waves. If the merger product has a mass larger than the Chandrasekhar limit, it may explode as a Type Ia supernova. This is the Double Degenerate (DD) model for Type Ia supernovae (however, see Saio \& Nomoto 1985, 2004, for the alternative view that a DD merger produces a neutron star). I will briefly discuss the merits of the SD and DD models in Section 2.

In binaries in which the mass-receiving white dwarf is of the O-Ne-Mg type (these can under certain conditions be produced by stars with initial masses between $\sim 8$ and $12 \mathrm{M}_{\odot}$; see Miyaji et al. 1980; Podsiadlowski et al. 2004), mass transfer to the star until it reaches the Chandrasekhar limit will lead to the collapse of the star due to the capture of degenerate electrons by nuclei of $\mathrm{Ne}$ and Mg (e.g. Miyaji et al. 1980; Nomoto 1984; Canal, Isern \& Labay 1990; Pylyser \& Savonije 1988). The outcome of this Accretion-Induced Collapse (AIC) is expected to be a neutron star. (Under very special conditions a CO white dwarf might in some cases collapse to a neutron star, e.g. Canal, Isern \& Labay 1990). This is one way to produce neutron stars in binary systems, which may later evolve into X-ray binaries and binary radio pulsars. 
However, the majority of the neutron stars in X-ray binaries and binary radio pulsars are expected to have been produced by the direct core collapse of stars that started their lives with masses $\geq 8 \mathrm{M}_{\odot}$. Here there is still a difference between stars which started out with masses between $\sim 8$ and $12 \mathrm{M}_{\odot}$, in which a degenerate $\mathrm{O}-\mathrm{Ne}-\mathrm{Mg}$ core forms, which collapses as a result of electron capture (Miyaji et al. 1980; Podsiadlowski et al. 2004), and stars more massive than about $12 \mathrm{M}_{\odot}$, in which the core passes through all stages of nuclear fusion until a degenerate iron core forms which collapses to a neutron star.

In recent years it has become clear from the study of Be-type X-ray Binaries (Pfahl et al. 2002) and of binary radio pulsars (van den Heuvel 2004) that these two types of core collapses most probably produce neutron stars with different properties, i.e. with different masses and kick velocities (Podsiadlowski et al. 2004, 2005; van den Heuvel 2004; Dewi, Podsiadlowski \& Pols 2005; Schwab, Podsiadlowski \& Rappaport 2010).

In Section 3, I discuss in more detail these relatively new findings that appear to confirm that there are two different mechanisms by which neutron stars can form, as was originally suggested by Miyaji et al. (1980). I also briefly discuss there the role that AIC may play in the formation of neutron stars in globular clusters and in Low-Mass X-ray Binaries and binary pulsars observed in the Galactic disk.

\section{Type Ia supernova scenarios}

\subsection{The Single Degenerate model and its problems}

In the SD model the white dwarf is growing in mass due to the accretion of matter from its nondegenerate companion star. The problem here is that for a wide range of mass-transfer rates, the hydrogen accumulated on the surface of the white dwarf tends to ignite explosively, once the mass of the accreted layer exceeds a threshold value. Such thermonuclear explosions of the accreted hydrogen layer are observed as various types of nova outbursts, and it is quite possible that in many of these explosions much, if not all, of the accreted matter is ejected, such that little or no net-growth of the white dwarf may take place. The critical mass $\Delta \mathrm{M}_{\mathrm{c}}$ at which nuclear burning ignites decreases with increasing white dwarf mass and increasing accretion rate (e.g. Nomoto 1982a; Prialnik \& Kovetz 1995; Townsley \& Bildsten 2005).

Fig. 1 (from Townsley \& Bildsten 2005) depicts the various nuclear burning regimes, of hydrogen-rich matter (70 per cent hydrogen), on the surface of a white dwarf, as a function of accretion rate and white dwarf mass. For a small range of accretion rates, $(1-4) \times 10^{-7} \mathrm{M}_{\odot} / \mathrm{yr}$, indicated by the hatched band in the figure, the hydrogen burns steadily on the surface of the white dwarf, and for these accretion rates the white dwarf will be able to steadily grow in mass. Below this range of accretion rates, the burning takes place in flashes. The curves in the figure depict the critical masses $\Delta \mathrm{M}_{\mathrm{c}}$ of the accreted hydrogen layer at the moment at which burning is ignited. The accretion rate onto the WD determines the strength of the outburst. Higher accretion rates lead to less violent outbursts. 


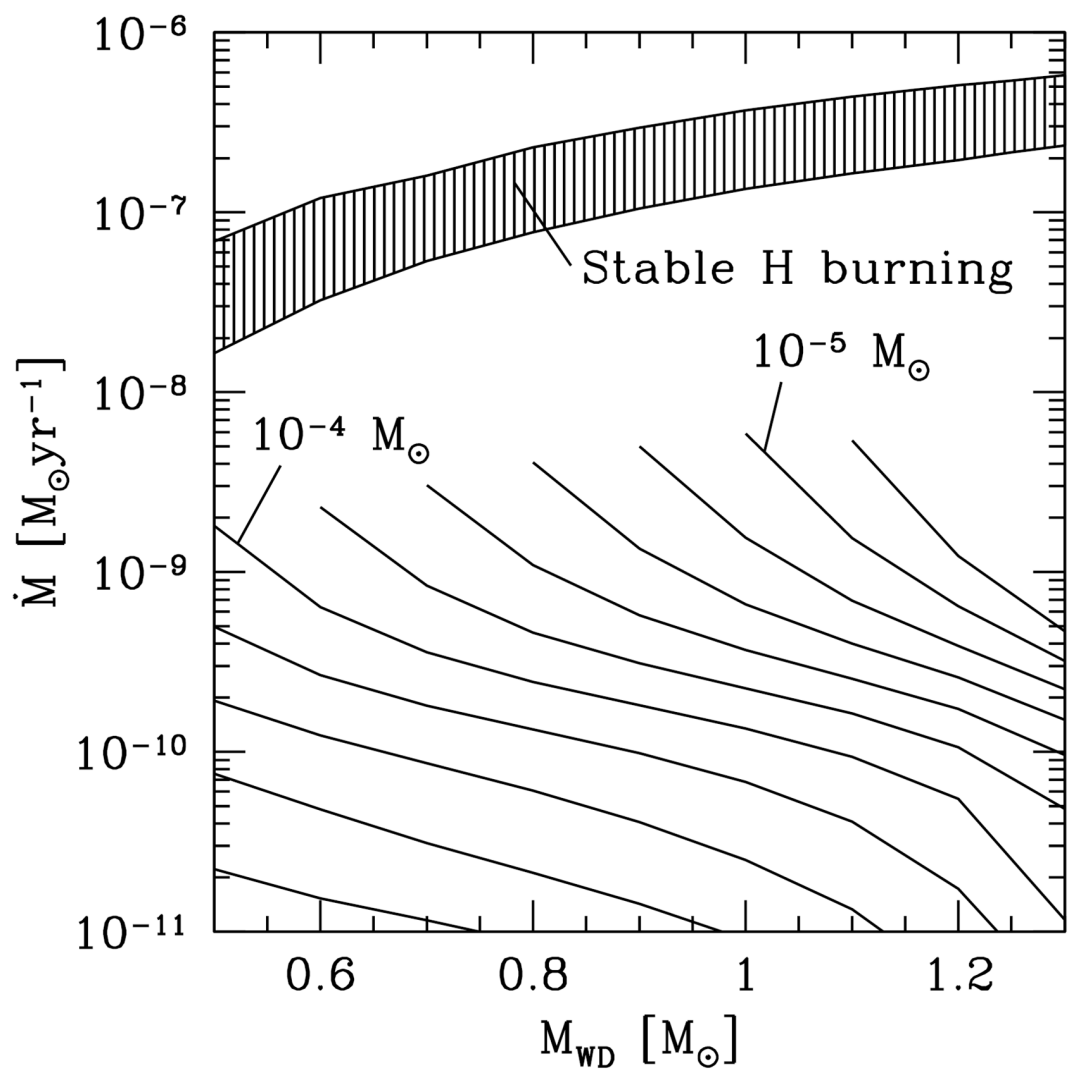

Figure 1. Hydrogen ignition masses $\Delta \mathrm{M}_{\mathrm{ign}}$ for $\mathrm{CO}$ white dwarfs that have reached their equilibrium core temperatures. Contours are equally spaced in ignition masses, labels indicate $\Delta \mathrm{M}_{\mathrm{ign}}$ in $\mathrm{M}_{\odot}$. The vertically hatched region indicates where steady burning of $\mathrm{H}$ is expected (Nomoto 1982a). At higher mass-accretion rates either envelope build-up and expansion into a giant, or the development of a strong wind is expected (after Townsley \& Bildsten 2005).

For very high accretion rates, above twice the maximum accretion rate for steady nuclear burning, the burning is still steady, but due to the super-Eddington energy generation by this burning, a strong stellar wind develops in which the excess accreted matter is blown away. For these accretion rates, the WD still grows, but less efficiently than in the accetion range for steady burning without a wind, since part of the transferred matter will be lost, and cannot contribute to the growth of the WD.

Binaries in which steady nuclear burning on the surface of the WD takes place were identified with the bright Super Soft X-ray Sources (SSS) discovered by the ROSAT satellite (van den Heuvel et al. 1992; see the reviews by Rappaport \& Di Stefano 1996 and Kahabka \& van den Heuvel 1997, 2006). These typically emit of order $10^{38} \mathrm{ergs} / \mathrm{s}$ in the form of very soft X-rays 
peaking in the energy range $20-100 \mathrm{eV}$. (It should be remembered that, contrary to the case of a neutron star, in the case of a WD accretion of matter onto the surface produces far less energy that nuclear burning, so the main energy source of the SSS is nuclear burning, not accretion; see also Kahabka, van den Heuvel \& Rappaport 1999).

Although some symbiotic binaries and old novae also appear as SSS, the main group of SSS is that of the so-called 'classical' ones, which are binaries with orbital periods of one day to a few days, in which a donor star in the mass range 1.5-2.5 $\mathrm{M}_{\odot}$ is transferring mass to the white dwarf on a thermal timescale of the donor. This yields typical mass-transfer rates of order $10^{-7}$ $\mathbf{M}_{\odot}$ per year. [These binaries are the higher-donor-mass analogues of the Cataclysmic Variables, and formed in the same way, through a phase of Common-Envelope evolution, starting from a wide binary consisting of a red giant with a degenerate core, plus an unevolved companion star of 1.5 to $2.5 \mathrm{M}_{\odot}$ (e.g. Rappaport \& Di Stefano 1996; Kahabka \& van den Heuvel 1997)]. These classical SSS are an interesting subgroup of potential Type Ia SN progenitor candidates (e.g. Di Stefano 2010).

WDs with mass accretion rates below the range for steady burning, but still quite high, e.g. in the range between $10^{-8.5}$ and $10^{-7} \mathrm{M}_{\odot}$ per year, have only weak flashes of nuclear burning, such that most of the accreted matter may be retained. These systems, which may appear as various types of novae, may also contribute considerably to the Type Ia SN rate. This is the regime of accretion for observed recurrent novae such as RS Ophiuchi and T Corona Borealis, which are binaries composed of a solar-mass red-giant which fills its Roche lobe plus a massive WD $\left(\mathrm{M} \sim 1.0\right.$ to $\left.1.2 \mathrm{M}_{\odot}\right)$. They erupt every few decades and with an average accretion rate of order $10^{-7.5} \mathrm{M}_{\odot} / \mathrm{yr}$, they need $\sim 10^{7} \mathrm{yr}$ to accrete the $\sim 0.3 \mathrm{M}_{\odot}$ needed to reach the Chandrasekhar limit. So, if these WDs are composed of $\mathrm{C}$ and $\mathrm{O}$, they are excellent candidates for producing a Type Ia SN (Bildsten 2010).

Other suggested candidates for the hydrogen SD model are the symbiotic binaries, some of which are also SSS. These are wide binaries consisting of a red giant that does not fill its Roche lobe but has a strong stellar wind, and a WD that is accreting matter from this wind. As wind accretion is relatively inefficient and the lifetimes of the red giants are limited, it is questionable if many of the WDs in symbiotic binaries will ever be able to grow to the Chandrasekhar limit.

An interesting other type of SD model is one in which the donor star is a helium star. Such systems are, like the DD systems, the results of two CE phases. These systems were recognized as potentially interesting Type Ia SN candidate progenitors (e.g. Yungelson 2005 and references therein), which has been confirmed by later simulations (e.g. Wang \& Han 2010). Here the accreted helium layer detonates and sends in a shockwave which may ignite carbon close to the centre of the white dwarf, triggering a Type Ia supernova. The mass of the white dwarf may in this case also be below the Chandrasekhar limit (e.g. Yungelson 2005; Bildsten 2010). Along similar lines, several authors have recently suggested that all Type Ia SNe might result from explosions of sub-Chandrasekhar-mass WDs (e.g. see Ruiter, Belczynski \& Fryer 2009; Ruiter et al. 2010, and references given therein). However, since it remains to be seen whether these can indeed 
produce 'standard candle-like' explosions, as expected from WDs which all explode at the same Chandrasekhar mass, I do not further discuss these models here.

\subsection{The relative importance of the SD and the DD scenarios for the Type Ia $\mathrm{SN}$ rate in galaxies}

In order to examine the relative contributions of the SD and DD models to the Type Ia SN rates in different galaxies, several groups have carried out population synthesis evolution calculations assuming a realistic initial fraction of binary systems (usually between 50 and 100 per cent). Among the SD models one still has to distinguish between SD systems in which the donor is a hydrogen-rich star (SD,H) and one in which it is a helium-burning helium star (SD,He).

In such calculations one starts from a burst of star formation, and follows the evolution of the entire population of this starburst in the course of time, including the evolution of all the types of binary systems. For the starburst one assumes a distribution of the stellar masses (which includes the primary stars of binaries) according to the Initial Mass Function (IMF). The binaries are assigned an orbital semi-major axis and a mass ratio, taken from the observed distributions of these binary parameters (e.g. van den Heuvel 1994). One then has to use a binary evolution code in which the evolution of all types of binaries, with different initial primary star masses, orbital radii (orbits are mostly assumed to be circular) and mass ratios are included.

Here, a number of assumptions have to be made about what happens in certain stages in the evolution of binaries for which the outcome is presently still (very) uncertain. These are particularly the stages in which a binary loses much mass and orbital angular momentum, for example in the very important Common Envelope phases. The outcome of Common Envelope evolution depends critically on what prescription for the energy and angular momentum losses during this phase is assumed, and different authors use here different formalisms, and different values for the 'CE-efficiency' parameter $\alpha_{\mathrm{ce}}$ for Common-Envelope evolution, which can give widely different results. This may, after two Common Envelope phases, lead to final orbital dimensions that differ by more than an order of magnitude. It appears that despite using widely different binary evolution and stellar evolution codes, the results obtained by different authors, although numerically quite different, show similar global trends for the predicted SD and DD Type Ia rates as a function of time following the starburst. As an illustration, Figs. 2 and 3 show the results obtained by the Moscow group (Yungelson 2005) and by Claeys et al. (2010), respectively. One observes in both cases that the DD model starts with a high rate some $(3-$ 7) $\times 10^{7} \mathrm{yr}$ after the starburst, and then decays for a Hubble time following roughly a $1 / \mathrm{t}$ relation. The reason for this $1 / \mathrm{t}$ behaviour of the DD rate is well understood (e.g. Lipunov, Panchenko \& Pruzhinskaya 2011; Moaz 2010).

On the other hand, for the simulations by Yungelson (2005), the (SD,He) model shows a broad peak between $4 \times 10^{7} \mathrm{yr}$ and about $10^{9} \mathrm{yr}$, which, during part of this time, can slightly exceed the DD rate. However, in the ( $\mathrm{SD}, \mathrm{He}$ ) explosions, Yungelson also included subChandrasekhar mass explosions, by assuming that after $0.15 \mathrm{M}_{\odot}$ has been accreted also a sub- 


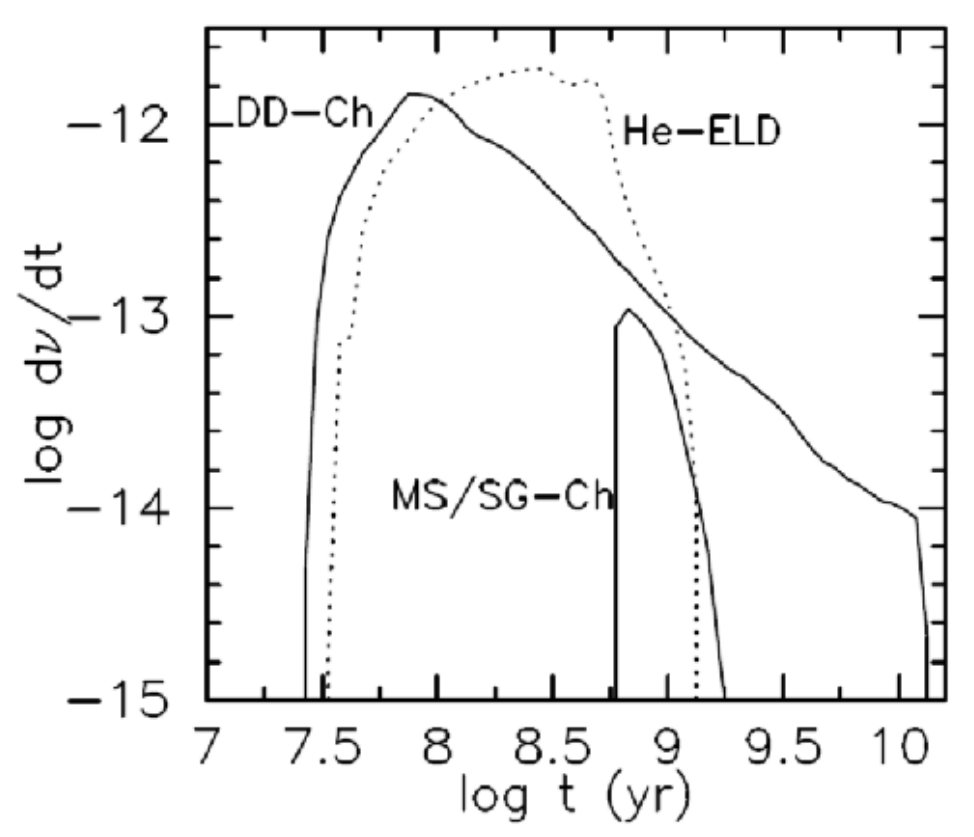

Figure 2. Rates of potential SNIa-scale events after a one year long burst of star formation that produces one solar mass of binary systems, after Yungelson (2005). The Helium-Edge-Lit Detonations include subChandrasekhar-mass events. If these are excluded, the explosions of helium-accretors terminate about 200 million years after the burst of star formation. The MS/SG-Ch systems are the Single-Degenerate $\mathrm{H}$-accretors (SuperSoft X-ray Sources), exploding at Chandrasekhar mass.

Chandra white dwarf would, after an edge-lit He-explosion, ignite C-burning close to its centre. Whether this will really occur is uncertain. If one excludes the sub-Chandra cases, the (SD, He) explosions in Yungelson's model terminate at $\sim 2 \times 10^{8} \mathrm{yr}$, and this is also the case in the Claeys et al. model (see Fig. 3), and the same is true for the simulation of Wang \& Han (2010) . In Yungelson's simulation the $(\mathrm{SD}, \mathrm{H})$ rate begins to rise at about $6 \times 10^{8} \mathrm{yr}$ and cuts off at $2 \times 10^{9}$ years. On the other hand, in the Claeys et al. simulation with $\alpha_{\mathrm{ce}}=1$, the $(\mathrm{SD}, \mathrm{H})$ model begins to contribute already at $10^{8} \mathrm{yr}$, and reaches values similar to the DD rate and then, like the DD rate, decays as $1 / \mathrm{t}$ for a Hubble time. One thus sees that only the behaviour of the $(\mathrm{SD}, \mathrm{H})$ rate is very different between the simulations of Figs. 2 and 3, due to quite different assumptions concerning the behaviour of the accreting white dwarfs for $(\mathrm{SD}, \mathrm{H})$ case. Other simulations, such as those by Mennekens et al. (2010a,b), Ruiter, Belczynski \& Fryer (2009) and Ruiter et al. (2010) show a similar behaviour for the DD model, but for the two SD models, differences can be quite considerable.

Observations of the 'delay rates', i.e. the change of the Type Ia SN rate as a function of time, in elliptical galaxies (that have not had star formation for several billions of years), show 


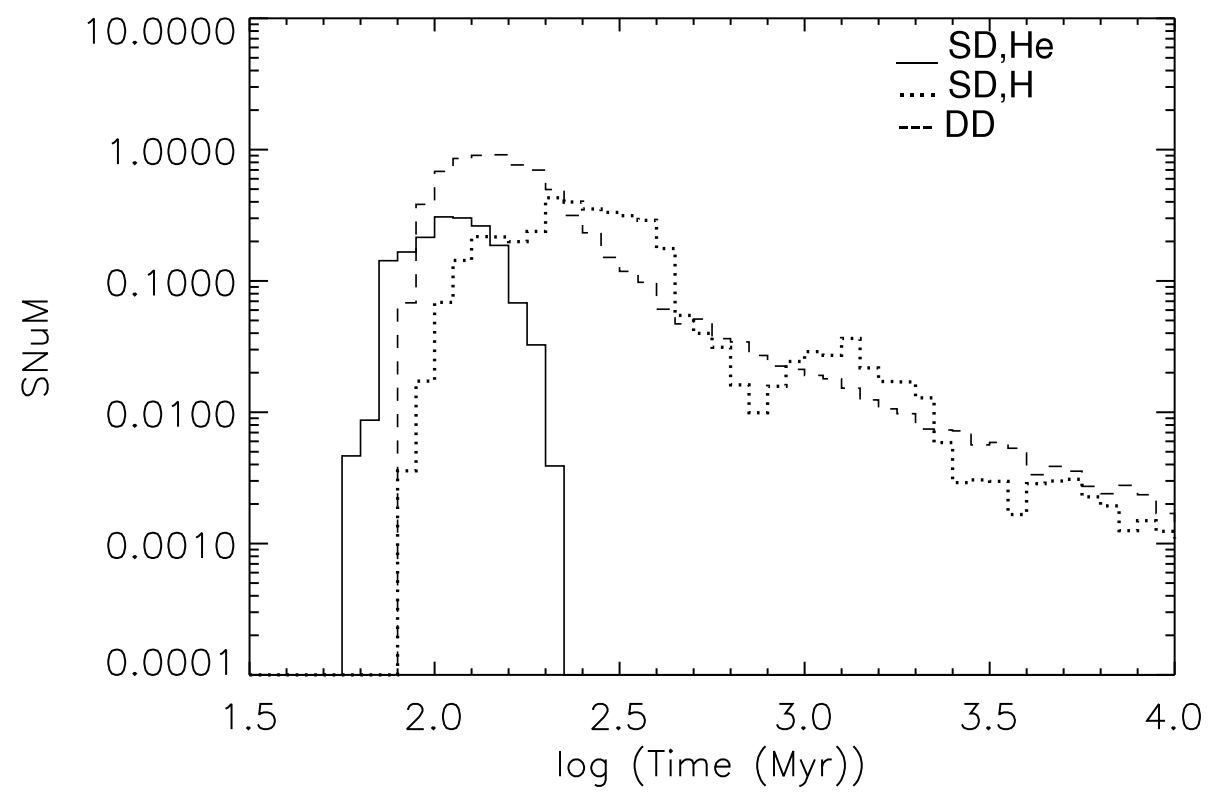

Figure 3. Type Ia SN event rates as a function of time after a single burst of star formation, for the three different progenitor scenarios indicated in the figure, as calculated by Claeys et al. (2010), assuming an efficiency parameter for Common-Envelope evolution $\alpha_{\mathrm{ce}}=1$.

that these follow a 1/t behavior (Totani et al. 2008; Moaz 2010), which appears to suggest that in elliptical galaxies the DD process dominates (although in the simulations Claeys et al. this could still be due to the $(\mathrm{SD}, \mathrm{H})$ model). For a detailed discussion of the results obtained by different authors, I refer to Nelemans (2010).

One observes from Figs. 2 and 3 that when the starburst is still young (from $(3-4) \times 10^{7} \mathrm{yr}$ on) the $(\mathrm{SD}, \mathrm{He})$ and $\mathrm{DD}$ processes dominate, and only after $(1-6) \times 10^{8} \mathrm{yr}$ the $(\mathrm{SD}, \mathrm{H})$ process begins to kick in, and then may be quite important for at least a few billion years. One also sees in these figures, that the absolute Type Ia rate predicted by these models is very high at young ages. Therefore, all models predict a much higher Type Ia SN rate for star-forming galaxies such as spirals and irregulars, than for elliptical galaxies. This fits very well with the observations, as it is well known that the Type Ia SN rate observed in star-forming galaxies is much higher than that in ellipticals (e.g. Garnavich 2010). The difference between the star-forming galaxies and the ellipticals is, apart form these different SN Ia rates, that the sole type of SNe found in ellipticals is the SN Ia, while in star-forming galaxies one also finds the core-collapse types of SNe: the Types II, Ib and Ic.

The conclusions from these population synthesis calculations are as follows. 
(i) Their global predictions are in agreement with the observed trends of the evolution of the Type Ia SN rate with time in galaxies, and particularly,

(ii) the $1 /$ t behaviour of the Type Ia time-delay curve observed in elliptical galaxies fits well with the predictions for the DD process obtained by all authors.

(iii) The much higher observed Type Ia SN rates in star-forming galaxies are well predicted by all models.

(iv) At early times $\left(3 \times 10^{7}\right.$ to $2 \times 10^{8}$ yr) the Type Ia SN rate is expected to be dominated by the $(\mathrm{SD}, \mathrm{He})$ and the $\mathrm{DD}$ processes;

(v) while at middle ages $(0.2-2) \times 10^{9}$ yr the Type Ia SN are expected to be produced by a mix of the $(\mathrm{SD}, \mathrm{H}),(\mathrm{SD}, \mathrm{He})$ and $\mathrm{DD}$ processes.

\section{Formation processes for neutron stars in binaries: evidence for two different NS formation mechanisms, yielding different neutron star masses and kick velocities}

\subsection{Two classes of B-emission X-ray binaries}

A very important discovery by Pfahl et al. (2002) was that there are two distinct classes of Bemission/neutron star systems (X-ray binaries as well as binary radio pulsars): one with orbits of small eccentricity $(<0.25)$, in which the neutron star received hardly any velocity kick at birth, and a class with substantial orbital eccentricities ( 0.3 to 0.9 ) in which the neutron stars must have received a kick velocity of several hundred $\mathrm{km} / \mathrm{s}$ at birth. A B-emission X-ray binary is a High Mass X-ray Binary (HMXB) consisting of a neutron star plus a rapidly rotating early B-type star, with a mass typically of $\sim 8$ to $20 \mathrm{M}_{\odot}$ and an orbital period between about 15 days and several years. The class with low orbital eccentricities (low birth kicks) is substantial and may comprise the majority of all Be/X-ray binaries (Pfahl et al. 2002). This may be partly a 'selection effect', as a considerable fraction of the high-kick systems may have been disrupted at the birth of the neutron star.

\subsection{Double neutron stars and their formation history: evidence that low kick velocities are related to low neutron star masses}

Double-neutron-star systems tend to have very narrow orbits (see Table 1) and are the later evolutionary products of wide high-mass X-ray binary systems with orbital periods $>100$ days (van den Heuvel \& Taam 1984; Bhattacharya \& van den Heuvel 1991), which are mostly B-emission $\mathrm{X}$-ray binaries. When the massive star in such a system has expanded to become a red giant, its envelope engulfs the neutron star, causing this star to spiral down into this envelope, reducing its orbital separation by several orders of magnitude. The large energy release due to friction 
and accretion during this spiral-in process is expected to cause the hydrogen-rich envelope of the giant to be expelled such that a very close binary remains, consisting of the helium core of the giant together with the neutron star. (Depending on the orbital separation at the onset of spiral-in, the helium core itself may already be (somewhat) evolved and possibly already have some $\mathrm{C}$ and $\mathrm{O}$ in its core). Due to the large frictional and tidal effects during spiral-in, the orbit of the system is expected to be perfectly circular. The helium star that remains after the spiral-in generates its luminosity by helium burning, which produces $\mathrm{C}$ and $\mathrm{O}$, and subsequently by carbon burning, produces $\mathrm{Ne}$ and $\mathrm{Mg}$.

If the helium star has a mass in the range 1.6 to $\sim 2.8 \mathrm{M}_{\odot}$ (corresponding to a main-sequence progenitor in the range of 8 to $12( \pm 1) \mathrm{M}_{\odot}$; the precise limits of this mass range depend on metallicity and on the assumed model for convective energy transport; Podsiadlowski et al. 2004 ), it will, during carbon burning, develop a degenerate $\mathrm{O}-\mathrm{Ne}-\mathrm{Mg}$ core, surrounded by episodic Cand He-burning shells (Nomoto 1984; Habets 1986). When such a degenerate core develops, the envelope of the helium star begins to expand, causing the onset of mass transfer by Roche-lobe overflow in a binary system (Habets 1986; Dewi \& Pols 2003). Roche-lobe overflow leads to the formation of an accretion disk around the neutron star and accretion of matter with angular momentum from this disk will cause the spin frequency of the neutron star to increase. Therefore one expects during the later evolution of these helium stars of relatively low mass, the first-born neutron star in the system to be 'spun up' to a short spin period. This neutron star had already a long history of accretion: first when it was in a wide binary with an early-type (presumably Be) companion; subsequently during the spiral-in phase into the envelope of its companion and now as companion of a Roche-lobe overflowing helium star. Since all binary pulsars which had a history of mass accretion (the so-called 'recycled' pulsars; Radhakrishnan \& Srinivasan $1982,1984)$ tend to have much weaker magnetic fields than normal single pulsars, it is thought that accretion in some way causes a weakening of the surface dipole magnetic field of neutron stars (Taam \& van den Heuvel 1986). Several theories have been put forward to explain this accretion-induced field decay (e.g. Bhattacharya \& Srinivasan 1995; Zhang 1998; Cumming, Arras \& Zweibel 2004). With a field weakened to about $10^{10}$ Gauss (as observed in the recycled components of the double neutron stars (see Table 1), and an Eddington-limited accretion rate of helium $\left(4 \times 10^{-8} \mathrm{M}_{\odot} / \mathrm{yr}\right)$ a neutron star can be spun-up to a shortest possible spin period of a few tens of milliseconds (Smarr \& Blandford 1976; Bhattacharya \& van den Heuvel 1991). [If one assumes that spin-up requires Roche-lobe overflow, such spin-up will not take place if the helium star is more massive than about $3.5 \mathrm{M}_{\odot}$, because these stars do not greatly expand during their later evolution and therefore do not go through a sufficiently long-lasting phase of Roche-lobe overflow].

When the helium star finally explodes as a supernova, the second neutron star in the system is born. This is a newborn neutron star without a history of accretion and is therefore expected to resemble the 'normal' strong-magnetic field single radio pulsars (Srinivasan \& van den Heuvel 1982), which have typical surface dipole magnetic fields strengths of $10^{12}-10^{13}$ Gauss. This theoretical expectation has been confirmed by the discovery of the double pulsar systems PSR $\mathrm{J} 0737-3039 \mathrm{AB}$, which consists of a recycled pulsar ( $\operatorname{star} \mathrm{A}$ ) with a very rapid spin ( $\mathrm{P}=23 \mathrm{~ms}$ ) and a weak magnetic field $\left(7 \times 10^{9} \mathrm{G}\right)$ and a normal strong-magnetic-field $\left(6 \times 10^{12} \mathrm{G}\right)$ pulsar 
(star B), with a 'normal' pulse period of $2.8 \mathrm{sec}$ (Burgay et al. 2003; Lyne et al. 2004; see Table 1). The explosive mass loss in the second supernova has made the orbit eccentric and since the two neutron stars are basically point masses, tidal effects in double neutron star systems will be negligible and there will be no tidal circularization of the orbit. (On timescales of tens of millions of years the orbits may be circularized by a few tens of per cent due to the emission of gravitational waves in the shortest-period system of PSR J0737-3039; assuming the observed pulsars to have an age of order half their spin down timescale, this is a negligible effect in all other systems, except in the final stages of spiraling together; e.g. Shapiro \& Teukolsky 1983).

\subsection{A correlation between a small velocity kick and a low mass of the neutron star in double neutron star systems}

In case of spherically symmetric mass ejection in the supernova explosion there is a simple relation between the orbital eccentricity and the amount of mass $\Delta M_{S N}$ ejected in the supernova:

$$
e=\frac{\Delta M_{S N}}{M_{n s 1}+M_{n s 2}}
$$

where $M_{n s 1}$ and $M_{n s 2}$ are the masses of the first- and the second-born neutron stars. I made calculations of the effect of the supernova mass loss plus a kick of $400 \mathrm{~km} / \mathrm{s}$, as observed for the young single pulsars (Hobbs et al. 2005) on the final orbital eccentricity of a double neutron star. I chose as a representative progenitor system: a binary with a circular orbit and a period of 4.8 $\mathrm{hr}$, consisting of a $2 \mathrm{M}_{\odot}$ helium star plus a $1.38 \mathrm{M}_{\odot}$ neutron star, and assumed the helium star to leave a $1.25 \mathrm{M}_{\odot}$ neutron star, which received a randomly directed kick at birth of $400 \mathrm{~km} / \mathrm{s}$. From these calculations it was found that about half of all systems is disrupted by the explosion and that the systems that remain bound have on average an orbital eccentricity $>0.7$.

However, Table 1 shows that five out of the eight double neutron star systems known in the Galactic disk have eccentricities below 0.25. Taking into account that the sudden massloss effects of the supernova also induced an orbital eccentricity, this unusually large fraction of low-eccentricity systems strongly suggests that the second-born neutron stars in these systems received at most only a very small velocity kick at their births. This is further confirmed by the fact that, as Dewi, Podsiadlowski \& Pols (2005) have shown, the observed correlation between the orbital eccentricity and the spin-period of the recycled neutron stars in the double neutron star systems (Faulkner et al. 2005) can be explained only if the second-born neutron stars received hardly any kick velocity at their birth (less than a few tens of $\mathrm{km} / \mathrm{sec}$ ). Indeed, Faulkner et al. had already pointed out that this relation could be understood on the basis of equation (1) if no kick had been imparted at birth to the second-born neutron star. It thus appears that, as pointed out by van den Heuvel (2004), these second-born neutron stars belong to the same 'kick-less' class as the neutron stars in the low-eccentricity class of Be/X-ray binaries (Pfahl et al. 2002).

The same holds for the young strong-magnetic-field pulsar in the eccentric radio-pulsar bi- 
nary PSR J1145-6545 which has a massive white dwarf as a companion (Kaspi et al. 2000; Bailes et al. 2003; Bhat, Bailes \& Verbiest 2008). The orbital eccentricity of 0.172 of this binary shows that the neutron star was the last-born object in the system, since formation of a white dwarf as the second-born object in the system cannot induce an orbital eccentricity (Kaspi et al. 2000; Bailes et al. 2003, van den Heuvel 2004; Bhat et al. 2008). The low value of its eccentricity would be hard to understand if the neutron star received the canonical $400 \mathrm{~km} / \mathrm{s}$ kick (Hobbs et al. 2005) at its birth.

In the eccentric white-dwarf neutron-star system of PSR J1145-6545 the mass of the neutron star is known from the measurement of relativistic effects (periastron advance and Shapiro delay) to be $1.27( \pm 0.01) \mathrm{M}_{\odot}$ (Bhat et al.2008; number within parentheses indicates the $95 \%$ confidence boundary). Also in three of the other low-eccentricity double neutron stars the masses of both stars are accurately known from the measured relativistic effects.

(i) In PSR J0737-3039 the second-born neutron star has MB=1.2489( \pm 0.0007$)$ and the firstborn one has $\mathrm{MA}=1.3381( \pm 0.0007) \mathrm{M}_{\odot}($ Kramer et al. 2006).

(ii) In PSR J1756-2251 the second-born neutron star has a mass of $1.24( \pm 0.02)$ and the firstborn one a mass of 1.32( \pm 0.02$) \mathrm{M}_{\odot}$ (Stairs 2008).

(iii) In PSR J1906+0746 we observe the second-born (non-recycled) pulsar, which has a mass of $1.248( \pm 0.018) \mathrm{M}_{\odot}$, while its (invisible) recycled companion has a mass of $1.365( \pm$ $0.018) \mathrm{M}_{\odot}$ (Kasian 2008). The observed second-born pulsar here is very young $\left(\sim 10^{5} \mathrm{yr}\right)$ and is spinning fast $(\mathrm{P}=0.144 \mathrm{sec})$

In the other double neutron stars the masses of the stars are not yet accurately known, but in the two other low-eccentricity systems the second-born neutron stars must be less massive than $1.30 \mathrm{M}_{\odot}$ for the following reasons. In all double neutron star systems the relativistic parameter that can be measured most easily is the general relativistic rate of periastron advance, which directly yields the sum of the masses of the two neutron stars (e.g. Stairs 2004). In the systems of PSR J1518+4904 and PSR J1829+2456 the resulting sum of the masses turns out to be 2.62( \pm $0.07) \mathrm{M}_{\odot}$ (Nice, Sayer \& Taylor 1996) and 2.53( \pm 0.10$) \mathrm{M}_{\odot}$ (Champion et al. 2004), respectively. The individual masses of the neutron stars in these systems are still rather poorly determined, but in both these systems the already crudely determined other relativistic parameters indicate that the second-born neutron star has the lowest mass of the two (Stairs 2004). As in all these systems the sum of the masses is around $2.60 \mathrm{M}_{\odot}$, the second-born neutron stars in these systems cannot be more massive than $1.30 \mathrm{M}_{\odot}$.

One thus observes that in the six systems (out of seven, if J1145-6545 is included) with low orbital eccentricities the second-born neutron star has a low mass, close to $1.25 \mathrm{M}_{\odot}$ and belongs to the low-kick category. This is strong evidence that no (or a low) kick velocity is correlated with a low neutron star mass of around 1.25( \pm 0.05$) \mathrm{M}_{\odot}$ (see also Schwab 2010).

A neutron star mass of $\sim 1.25 \mathrm{M}_{\odot}$ corresponds to a pre-collapse mass of about $1.44 \mathrm{M}_{\odot}$ as 
during the collapse the gravitational binding energy of the neutron star of about $0.20 \mathrm{M}_{\odot}$ (slightly depending on the assumed equation of state of neutronized matter) is lost in the form of neutrinos. So apparently the cores which collapsed to form these second-born neutron stars had a mass very close to the Chandrasekhar mass.

\subsection{Formation mechanisms of neutron stars and possible resulting kicks}

There are two basically different ways in which neutron stars are expected to form (Miyaji et al. 1980; Canal et al. 1990).

(I) In stars which originated in the main-sequence with mass in the range between 8 and about $12( \pm 1) \mathrm{M}_{\odot}$, and which are in binaries produce helium stars in the mass range 1.6 to 2.8 $\mathrm{M}_{\odot}$. The O-Ne-Mg core which forms during carbon burning becomes degenerate and when its mass approaches the Chandrasekhar mass, and electron captures on $\mathrm{Mg}$ and $\mathrm{Ne}$ cause the core to collapse to a neutron star. Since these stars did not reach oxygen and silicon burning, the baryonic mass of the neutron star, which forms in this way is expected to be purely determined by the mass of the collapsing degenerate core, which is the Chandrasekhar mass. The gravitational mass of this neutron star is then the Chandrasekhar mass minus the gravitational binding energy of the neutron star, which is about $0.20 \mathrm{M}_{\odot}$. Thus a neutron star with a mass of about $1.25 \mathrm{M}_{\odot}$ is expected.

(II) In stars initially more massive than $12( \pm 1) \mathrm{M}_{\odot}$, the $\mathrm{O}-\mathrm{Ne}-\mathrm{Mg}$ core does not become degenerate and these cores proceed through oxygen and silicon burning to form an iron core. When the mass of this iron core exceeds the Chandrasekhar limit it collapses to form a neutron star. The precise way in which neutrino transport during core bounce and shock formation results in a supernova explosion is not yet fully understood. It appears that first the shock stalls and then several hundreds of milliseconds later is revitalized. Some fall back of matter from the layers surrounding the proto neutron star is expected to occur (e.g. Fryer 2004) such that the neutron star that forms may be substantially more massive than the baryonic mass of the collapsing Fecore.

The fact that the pre-collapse masses of the low-mass, low-kick neutron stars were very close to the Chandrasekhar limit suggests that these neutron stars are the result of the electron-capture collapse of the degenerate $\mathrm{O}-\mathrm{Ne}-\mathrm{Mg}$ cores of helium stars that originated in the mass range 1.6 to about $2.8 \mathrm{M}_{\odot}$ (initial main-sequence mass in the range 8 to about $12 \mathrm{M}_{\odot}$ ). Can one understand why such neutron stars would not receive a large birth kick whereas those formed by the collapse of an iron core would?

Burrows \& Hayes (1996) have pointed out that the violent large-scale convective motions in the core during $\mathrm{O}$ - and Si-burning just prior to the formation of the Fe-core may produce considerable large-scale density inhomogeneities in the mantle of the proto-neutron star. They showed that this may lead to asymmetric neutrino transport and escape, which may easily impart enough momentum to the neutron star to produce a space velocity of $500 \mathrm{~km} / \mathrm{s}$. Recent 3-D 
Table 1. Double neutron star binaries in the Galactic disk

\begin{tabular}{lccclllcl}
\hline $\begin{array}{l}\text { Pulsar } \\
\text { Name }\end{array}$ & $\begin{array}{c}\text { Spin } \\
\text { per. } \\
(\mathrm{ms})\end{array}$ & $\begin{array}{c}\mathrm{P}_{\text {orb }} \\
(\mathrm{d})\end{array}$ & $\mathrm{E}$ & $\begin{array}{l}\text { Mass } \\
\left(\mathrm{M}_{\odot}\right)\end{array}$ & $\begin{array}{l}\text { Mass } \\
\left(\mathrm{M}_{\odot}\right)\end{array}$ & $\begin{array}{l}\text { Sum of } \\
\text { masses } \\
\left(\mathrm{M}_{\odot}\right)\end{array}$ & $\begin{array}{c}\text { Bs } \\
\left(10^{10} \mathrm{G}\right)\end{array}$ & Ref \\
\hline J0737-3039A & 22.7 & 0.10 & 0.088 & $1.2489(7)$ & $1.3381(7)$ & $2.5870(3)$ & 0.7 & 1 \\
$\mathrm{~J} 0737-3039 \mathrm{~B}$ & 2770 & 0.10 & 0.088 & $1.3381(7)$ & $1.2489(7)$ & $2.5870(3)$ & $6 \times 10^{2}$ & 1 \\
$\mathrm{~J} 1518+4904$ & 40.9 & 8.63 & 0.249 & $1.05_{-0.11}^{+0.45}$ & $1.56_{-0.45}^{+0.13}$ & $2.62(7)$ & 0.1 & 2 \\
$\mathrm{~B} 1534+12$ & 37.9 & 0.42 & 0.274 & $1.3452(10)$ & $1.3332(10)$ & $2.678(1)$ & 1 & 3 \\
$\mathrm{~J} 1756-2251$ & 28.5 & 0.32 & 0.18 & $1.24(2)$ & $1.32(2)$ & $2.56(2)$ & 0.54 & 4 \\
$\mathrm{~J} 1811-1736$ & 104 & 18.8 & 0.828 & $1.11_{-0.15}^{+0.53}$ & $1.62_{-0.55}^{+0.52}$ & $2.60(10)$ & 1.3 & 3 \\
$\mathrm{~J} 1829+2456$ & 41.0 & 1.18 & 0.139 & $1.27_{-0.07}^{+0.11}$ & $1.30_{-0.05}^{+0.05}$ & $2.53(10)$ & $\sim 1$ & 5 \\
$\mathrm{~J} 1909-3744$ & 144 & 3.98 & 0.085 & $1.365(18)$ & $1.248(18)^{*}$ & $2.613(9)$ & 170 & 6 \\
$\mathrm{~B} 1913+16$ & 59 & 0.33 & 0.617 & $1.3873(3)$ & $1.4408(3)$ & $2.8281(1)$ & 2 & 4 \\
$\mathrm{~J} 1145-6545$ & 394 & 0.20 & 0.172 & $1.01(1) \mathrm{WD}$ & $1.27(1)$ & $2.28(1)$ & $10^{2}$ & 7 \\
\hline
\end{tabular}

References: (1) Kramer et al. (2006); (2) Nice et al. (1996); (3) Stairs (2004); (4) Stairs (2008); (5) Champion et al. (2004); (6) Kasian (2008); (7) Bhat et al. (2008).

* The observed pulsar here is the second-born non-recycled strong-magnetic field one.

numerical hydrodynamic core collapse and neutrino transport calculations by Scheck et al. (2004) and Arnett \& Maekin (2011) confirm this expectation. As no O- and Si-burning occur prior to the e-capture collapse of a degenerate $\mathrm{O}-\mathrm{Ne}-\mathrm{Mg}$ core, neutrino transport in this case may be close to spherically symmetric, leading to no (or a very small) kick velocity imparted during collapse, as was confirmed by detailed numerical hydrodynamical calculations of such stellar cores by Kitaura et al. (2006).

\subsection{Why are there no low-velocity, young, single radio pulsars?}

A detailed statistical study by Hobbs et al. (2005) of all available pulsar proper motions showed that the observed velocity distribution of single young (age $<3$ million years) radio pulsars is excellently represented by one single Maxwellian with a mean 3-D speed of about $400 \mathrm{~km} / \mathrm{sec}$, and that there is no evidence for a bimodal velocity distribution (viz.: a separate lower-velocity population of young single pulsars) as had been suggested earlier (e.g. Arzoumanian, Chernoff $\&$ Cordes 2002). In terms of the above-described model this would mean that single pulsars are solely the products of iron-core collapse supernovae, whereas neutron star formation by electroncapture collapse would occur only in interacting binaries.

This is indeed precisely what has been suggested by Podsiadlowski et al. (2004) on grounds of stellar evolution considerations. These authors argued that if stars in the mass range 8 to about $12 \mathrm{M}_{\odot}$ are single, they will later in life evolve towards the Asymptotic Giant Branch (AGB), where the convective envelope during 'dredge-up' will penetrate the evolved helium core (which 
on the AGB has a degenerate $\mathrm{O}-\mathrm{Ne}-\mathrm{Mg}$ central core) and during this phase will erode away the outer helium layers of this core down to the degenerate O-Ne-Mg core. This prevents the latter core from further growth towards the Chandrasekhar limit. Their suggestion is therefore that single stars in the mass range 8 to $12( \pm 1) \mathrm{M}_{\odot}$ do not evolve to core collapse, but after heavy mass loss on the AGB will leave $\mathrm{O}-\mathrm{Ne}-\mathrm{Mg}$ white dwarfs.

Single stars more massive than about $12( \pm 1) \mathrm{M}_{\odot}$ will not produce degenerate $\mathrm{O}-\mathrm{Ne}-\mathrm{Mg}$ cores and will, in this picture, evolve through O- and Si-burning towards an iron-core collapse supernova. According to the above-described model calculations of Scheck et al.(2004) and Arnett \& Maekin (2011) such a supernova presumably imparts a large kick velocity to the neutron star. Thus, combining these models with that of Podsiadlowski et al. (2004), one expects single pulsars to generally have received a large velocity kick at birth.

On the other hand, if the 8 to $12( \pm 1) \mathrm{M}_{\odot}$ star is in an interacting binary, the star cannot reach the AGB: before that time it already overflows its Roche lobe and loses its hydrogen-rich envelope by mass transfer towards its companion star (and in many cases, partly out of the system). For this reason in these systems a helium star in the mass range 1.6 to $2.8 \mathrm{M}_{\odot}$ will be left, which will produce a growing degenerate $\mathrm{O}-\mathrm{Ne}-\mathrm{Mg}$ core that evolves towards e-capture collapse. Thus, the e-capture collapse supernovae are, according to the model of Podsiadlowski et al. (2004), expected to solely occur in interacting binaries, and these will produce neutron stars of about $1.25 \mathrm{M}_{\odot}$.

These are then to be identified with the low-kick-velocity (low-mass) neutron stars that we observe in the double neutron star systems in Table 1 (van den Heuvel 2004; Podsiadlowski et al. 2005). One therefore would expect the low-kick low-mass neutron stars to solely be formed in interacting binaries, while single stars, or components of wide non-interacting binaries only produce high-kick-velocity neutron stars.

\subsection{Consequences for the occurrence of neutron star formation by Accretion-Induced Col- lapse (AIC)}

An important consequence of the above described model for the origins of kicks is that the accretion-induced collapse (AIC) of an O-Ne-Mg white dwarf in a close binary will not induce a sizeable kick velocity to the thus formed neutron star. Since in this process only the bindingenergy mass equivalent of a neutron star $\left(\sim 0.2 \mathrm{M}_{\odot}\right)$ is explosively lost, the mass-loss-induced runaway velocity of the resulting neutron-star binary is not expected to exceed a few tens of $\mathrm{km} / \mathrm{s}$ for systems with Cataclysmic-Variable-like dimensions prior to the AIC. Such systems are therefore unlikely to escape from globular clusters and it seems most plausible, in view of the very large white dwarf populations in these clusters, that AIC is the dominant neutron-star forming mechanism in such clusters. This at the same time would explain why in some globular clusters, despite their ages of over ten billion years, still apparently young strongly magnetized radio pulsars are present (Lyne, Manchester \& D'Amico 1996; an example is the strong-magentic-field globular cluster binary radio pulsar PSR B1719-19, with $P=1.0$ second, $B=10^{12} \mathrm{G}$ ). Although in 
globular clusters there is a large population of weakly magnetized millisecond pulsars, produced by accretion-driven binary recycling, the presence of already 3 short-lived strong-magnetic field radio pulsars in globular clusters implies that these have a birthrate that may be higher than that of the $\sim 100$ millisecond pulsars known in globular clusters, as the latter ones will live almost eternally (spindown timescales of Gigayears).

Furthermore, the existence of the very wide radio pulsar binaries with circular orbits, such as PSR B0820+02 (orbital period 3.5 years) so far was very puzzling, as at the onset of the mass transfer from the low-mass red-giant progenitor of the white dwarf companion of this pulsar, the orbital period of the system was already about one year (Verbunt \& van den Heuvel 1995). A neutron star that received a few hundred $\mathrm{km} / \mathrm{s} \mathrm{kick}$ at its birth could never have remained bound to a low-mass companion star in such a wide system. Formation from a white dwarf by a kickless AIC in a wide symbiotic-type binary seems a plausible way to solve this problem (although direct formation from the e-capture collapse of a helium core of $\sim 2 \mathrm{M}_{\odot}$ cannot be excluded).

\subsection{Massive neutron stars in binaries: a third type of neutron star?}

Since 1975 it has been known that the accreting neutron star in the eclipsing High Mass X-ray Binary Vela X-1 (4U0900-40) has a mass considerably larger than the Chandrasekhar limit. The best modern determination of the mass of this neutron star from the Doppler-measurements of the orbits of both stars in the system is 1.86( \pm 0.15$)$ solar masses (Barziv et al. 2001; Quaintrell et al. 2003). In view of the very short lifetime of HMXBs the growth in mass by accretion of this neutron star was negligible, hence this neutron star must have been born directly with a large mass.

Models of the final evolution of massive stars by Timmes, Woosley \& Weaver (1996) show that at an initial stellar mass of about $19 \mathrm{M}_{\odot}$ there occurs a jump in the mass of the collapsing iron core from 1.4 to $\sim 1.7 \mathrm{M}_{\odot}$. Hence, stars more massive than about $19 \mathrm{M}_{\odot}$, like the progenitor of the neutron star in Vela X-1, are expected to leave behind quite massive neutron stars in the range 1.8 to $2.0 \mathrm{M}_{\odot}$ (if a fall-back of a few tenths of a solar mass is included) or black holes, in the case of a large fall-back mass.

Interestingely, recently several new massive neutron stars have been discovered in binary radio pulsar systems: PSR J1614-2230 with a mass of $1.97 \pm 0.04 \mathrm{M}_{\odot}$ (Demorest et al. 2010) and PSR J1903+0327 with a mass of $1.667 \pm 0.021 \mathrm{M}_{\odot}$ (Freire et al. 2011). The latter pulsar is a millisecond one $(\mathrm{P}=2.15 \mathrm{~ms})$ and its high mass could be the result of a long-lasting accretion phase of a neutron star that started out with a mass $\sim 1.4 \mathrm{M}_{\odot}$. The same might be true for the 1.97 $\mathrm{M}_{\odot}$ neutron star PSR J1614-2230 ( $\left.\mathrm{P}=3.1 \mathrm{~ms}\right)$. This pulsar is in a relatively wide and circular orbit ( $\mathrm{P}=8.7$ days) with a $0.5 \mathrm{M}_{\odot} \mathrm{CO}$ white dwarf companion. Its short pulse period suggests that it has accreted at least $\sim 0.1 \mathrm{M}_{\odot}$. It could have formed directly with a high mass in core collapse, if its progenitor started out with a mass in excess of $19 \mathrm{M}_{\odot}$. It is not difficult to make models for such an origin of this system (e.g Lin et al.2010; Tauris, private communication). Alternatively, it might have started out with a mass of $\sim 1.4 \mathrm{M}_{\odot}$, but then it must have accreted some $0.6 \mathrm{M}_{\odot}$, 
which is a very large amount, but not impossible for a millisecond pulsar (e.g. van den Heuvel 1995). In the latter case, however, one wonders why it has not been spun up to a rotation period shorter than one millisecond. For this reason, it seems most likely to me that PSR J1614-2230 is the second example of a neutron star that was born already with a large mass, $\geq 1.70 \mathrm{M}_{\odot}$.

A third example is the compact star in the High Mass X-ray Binary 4U 1700-37. This compact star has a mass of $2.44 \pm 0.27 \mathrm{M}_{\odot}$, and has $0 \%$ probability to be $<1.60 \mathrm{M}_{\odot}$, and only $3.5 \%$ probability to be $<2.0 \mathrm{M}_{\odot}$ (Clark et al. 2002). It has never shown regular X-ray pulsations, but its X-ray spectrum is that of an accreting neutron star, which is significantly different from that of an accreting black hole (e.g. Clark et al. 2002). Its donor star is a highly luminous O6.5f supergiant star with a mass of $58 \pm 11 \mathrm{M}_{\odot}$, making it very likely that the progenitor of this compact star had a mass $>19 \mathrm{M}_{\odot}$. It therefore probably is the most massive neutron star known.

\subsection{Discussion; some further consequences of the model}

A consequence of the model in which neutron stars formed by electron-capture collapse receive hardly any kicks at birth, whereas those that are formed by Fe-core collapse receive large kicks, is that the formation of bound double neutron stars will be highly biased towards the lower-mass binaries, with components in the mass range 8 to about $12 \mathrm{M}_{\odot}$. The probability for disruption of such systems will be much lower than for systems in which the helium stars are above about $2.8 \mathrm{M}_{\odot}$. If the first supernova results from a helium star above $2.8 \mathrm{M}_{\odot}$, the resulting neutron star will get a large velocity kick, resulting in the formation of a high-eccentricity B-emission X-ray binary, or in the disruption of the system.

If the second helium star (resulting from the Be star in a Be/X-ray binary) has a mass above $2.8 \mathrm{M}_{\odot}$, it will again produce a high-kick neutron star, such that either the system is disrupted in the second supernova, or a very eccentric system results. As only three out of the eight double neutron stars in the Galactic disk have an orbital eccentricity larger than 0.25 , and since some of these eccentricities may also have resulted from the pure mass-loss effects of a helium star with a mass just below $2.8 \mathrm{M}_{\odot}$, the formation of double neutron stars from systems in which the last-born helium stars are more massive than $2.8 \mathrm{M}_{\odot}$ seems to rarely occur in nature.

\subsection{Summary}

There is strong observational evidence, in combination with predictions from stellar evolution theory, for the existence of three classes of neutron stars, with two different formation mechanisms: electron-capture collapse of degenerate $\mathrm{O}-\mathrm{Ne}-\mathrm{Mg}$ cores in stars in binaries with intitial masses between $\sim 8$ and $\sim 12 \mathrm{M}_{\odot}$, and iron-core collapse for all stars, single as well as binary, more massive than $\sim 12 \mathrm{M}_{\odot}$. 


\section{Acknowledgements}

I thank Gijs Nelemans, Lars Bildsten, Philip Podsiadlowski, Rosanne DiStefano and Thomas Tauris for many stimulating discussions on the physics and formation of compact stars and double neutron stars.

\section{References}

Arnett W.D., Maekin C., 2011, Preprint, University of Arizona, Steward Observatory

Arzoumanian Z., Chernoff D.F., Cordes J.M., 2002, ApJ, 568, 289

Bailes M., Ord S.M., Knight H., Hotan, A.W., 2003, ApJ, 595, L49

Barziv O., Kaper L., van Kerkwijk M.H., Telting J.H., van Paradijs J., 2001, A\&A, 377, 925

Bhat N.D.R., Bailes M., Verbiest J.P.W., 2008, Phys. Rev.D. 77, 124017

Bhattacharya D., Srinivasan G., 1995, in Lewin W.H.G., van Paradijs J., van den Heuvel E.P.J., eds, The Magnetic Fields of Neutron Stars and Their Evolution, X-ray Binaries, Cambridge Univ. Press, p. 495

Bhattacharya D., van den Heuvel E.P.J., 1991, Physics Reports, 203, 1

Bildsten L., 2010, Lorentz Center Workshop, Observational signatures of Type Ia supernova progenitors, Leiden, The Netherlands, September 20-24, 2010

Burgay M., et al., 2003, Nature, 426, 531

Burrows A., Hayes J., 1996, Phys. Rev. Letters, 76, 352

Canal R., Isern J., Labay J., 1990, ARA\&A, 28, 183

Champion D.J., Lorimer D.R., McLaughlin M.A. Cordes J.M., Arzoumanian Z., Weisberg J.M., Taylor, J.H., 2004, MNRAS, 350, L61

Claeys J.S.W., Pols O.R., Vink J., Izzard R.G., 2010, in Kalogera V., van der Sluys M., eds, International Conference on Binaries, AIP Conference Proceedings, New York, 1314, 262

Clark J.S., Goodwin S.P., Crowther P.A., Kaper L., Fairbairn M., Langer N., Brocksopp C., 2002, A\&A, 392, 909

Cumming A., Arras P., Zweibel E., 2004, ApJ, 609, 999

Demorest P.B., Pennucci T., Ransom S.M., Roberts M.S.E., Hessels, J.W.T., 2010, Nature, 467, 1081

Dewi J.D.M., Pols O.R., 2003, MNRAS, 344, 629

Dewi J.D.M., Podsiadlowski P., Pols, O.R., 2005, MNRAS, 363, L71

Di Stefano R. 2010, Lorentz Center Workshop, Observational signatures of Type Ia supernova progenitors, Leiden, The Netherlands, September 20-24, 2010

Faulkner A.J., et al., 2005, ApJ., 618, L119

Freire P.C.C, et al., 2011, MNRAS, 412, 2763

Fryer C.L., 2004, in Fryer C.L., ed, Stellar Collapse, Kluwer Acad. Publishers, Dordrecht

Garnavich P., 2010, Lorentz Center Workshop, Observational signatures of Type Ia supernova progenitors, Leiden, The Netherlands, September 20-24, 2010

Habets G.M.H.J., 1986, A\&A, 167, 61

Hobbs G., Lorimer D.R., Lyne A.G., Kramer M., 2005, MNRAS, 360, 3, 974

Hoyle F., Fowler W.A., 1960, ApJ, 132, 565

Iben I., Tutukov A.V., 1984, ApJ, Suppl. 54, 335

Kahabka P., van den Heuvel E.P.J., 1997, Annual Rev. Astron. Ap. 35, 69

Kahabka P., van den Heuvel E.P.J. 2006, in Lewin W.H.G., van der Klis M., eds, Compact Stellar X-ray Sources, Cambridge University Press, p. 461

Kahabka P., van den Heuvel E.P.J., Rappaport S.A., 1999, Scientific American, 280, 28 
Kasian L., 2008, in Bassa C., Wang Z., Cumming A., Kasspi V.M., eds, 40 years of Pulsars, Millisecond Pulsars, Magnetars and More, Am. Inst. of Phys. Conf. Series, 983, 369

Kaspi V.M., Lyne A.G., Manchester R.N., 2000, ApJ., 543, 1, 321

Kitaura F.S., Janka H.-Th., Muller E., 2006, A\&A, 450, 345

Kramer M., et al., 2006, Science, 314, 97

Lin J., Rappaport,IS.A., Podsiadlowski Ph., Nelson L., Paxton B., Todorov, P. 2010, arXiv: 1012.1877v1

Lipunov V.M., Panchenko I.E., Pruzhinskaya M.V. 2011, New Astronomy, 16, 250

Lyne A.G., Manchester R.N., D’Amico, N. 1996, ApJ 460, L41

Lyne A.G., et al., 2004, Science, 303, 5661, 1153

Mennekens N., van Beveren D., de Greve J.-P., De Donder, E., 2010a, A\&A, 515, A89

Mennekens N., van Beveren D., de Greve J.-P., De Donder, E., 2010b, in Kalogera V., van der Sluys M., eds, International Conference on Binaries, AIP Conference Proceedings, 1314, 239

Miyaji S., Nomoto K., Yokoi K., Sugimoto D., 1980, PASJ, 32, 303

Moaz D., 2010, in Kalogera V., van der Sluys M., eds, International Conference on Binaries, AIP Conference Proc., 1314, 223

Nelemans G., 2010, Lorentz Center Workshop, Observational signatures of Type Ia supernova progenitors, Leiden, The Netherlands September 20-24, 2010

Nice D.J., Sayer R.W., Taylor J.H., 1996, ApJ., 466, L87

Nomoto K., 1982a, ApJ 253, 798

Nomoto K., 1982b, ApJ 257, 780

Nomoto K., 1984, ApJ, 277, 791

Perlmutter S., et al., 1999, ApJ, 517, 565

Pfahl E., Rappaport S., Podsiadlowski P., Spruit H., 2002, ApJ, 574, 364

Phillips M.M., Lira P., Suntzeff N.B., Schommer R.A., Hamuy M., Maza J., 1999, AJ, 118, 1766

Podsiadlowski P., Langer N., Poelarends A.J.T., Rappaport S., Heger, A., Pfahl, E., 2004, ApJ, 612, 1044

Podsiadlowski P., Dewi, J.D.M., Lasaffre P., Miller J.C., Newton J.G., Stone, J.R., 2005, MNRAS, 361, 1243

Prialnik D., Kovetz A., 1995, ApJ 445, 789

Quaintrell H., Norton, A.J., Ash T.D.C., Roche P., Willems B., Bedding T.R., Baldry I.K., Fender R.P., 2003, A\&A, 401, 313

Pylyser E., Savonije G.J., 1988, A\&A, 191, 57

Radhakrishnan V., Srinivasan G., 1982, Current Science, 51, 1096

Radhakrishnan V., Srinivasan G., 1984, in Hidayat B., Feast M.W., 1981, eds, Proc 2nd Asian-Pacific Regional Meeting on Astronomy, IAU Bandung Indonesia, p. 423

Rappaport S., Di Stefano R., 1996, IAUS, 165, 415

Riess A.G., et al., 1998, AJ, 116, 1009

Ruiter A.J., Belczynski K., Fryer, C., 2009, ApJ, 699, 2026

Ruiter A.J., Belczynski K, Sim S.A., Hillebrandt W., Fink M., Kromer, M., 2010, in Kalogera V., van der Sluys M., eds, International Conference on Binaries, AIP Conference Proc, 1314, 233

Saio H., Nomoto, K., 1985, A\&A, 150, L21

Saio H., Nomoto, K., 2004, ApJ, 615, 444

Scheck L., Plewa T., Janka H.-T., Kifonidis K., Mueller, E., 2004, Phys. Rev. Letters, 92a, 1103

Schmidt B.P., et al, 1998, ApJ, 507, 46

Schwab J., Podsiadlowski Ph., Rappaport S., 2010, ApJ, 719, 722

Shapiro S.L., Teukolsky S.A., 1983, The physics of compact objects, New York, Wiley-Interscience, 663

Smarr L.L., Blandford R.D., 1976, ApJ, 207, 574

Srinivasan G., van den Heuvel E.P.J., 1982, A\&A, 108, 143

Stairs I.H., 2004, Science, 304, 547 
Stairs I.H., 2008, in C. Bassa, Z. Wang, A. Cumming, V.M. Kasspi, eds, 40 years of Pulsars, Millisecond Pulsars, Magnetars and More, Am. Inst. of Phys. Conf. Series, 983, 424

Taam R.E., van den Heuvel, E.P.J., 1986, ApJ, 305, 235

Timmes F.X, Woosley S.A., Weaver T.A. 1996, ApJ, 457, 834

Totani T., Morokuma T., Oda T., Doi M., Yasuda N., 2008, PASJ, 60, 1327

Townsley D.M., Bildsten L., 2005, ApJ, 628, 395

van den Heuvel E.P.J., 1994, in Shore S.N., Livio M., van den Heuvel E.P.J., eds, Interacting Binaries, Springer, Heidelberg, p. 263

van den Heuvel E.P.J., 1995, JA\&A, 16, 255

van den Heuvel E.P.J., 2004, in Schoenfelder V., Lichti G., Winkler C., eds, Proc. 5th INTEGRAL Workshop, ESA SP-552 (Noordwijk, ESA Publ.Div.ESTEC), p. 185

van den Heuvel, E.P.J., Taam, R. E., 1984, Nature, 309, 235

van den Heuvel E.P.J., Bhattacharya D., Nomoto K., Rappaport S.A., 1992, A\&A, 262, 97

Verbunt F., Van den Heuvel E.P.J., 1995, in Lewin W.H.G., van Paradijs J., van den Heuvel E.P.J., eds, X-ray Binaries, Cambridge Univ. Press, p. 457

Wang B., Han, Z., 2010, in Kalogera V., van der Sluys M., eds, International Conference on Binaries, AIP Conference Proc., 1314, 244

Webbink R.F., 1984, ApJ, 277, 355

Whelan J., Iben I., 1973, ApJ, 186, 1007

Yungelson L.R., 2005, in Sion E.M., Vennes S., Shipman H.L., eds, White dwarfs: cosmological and galactic probes, ASSL, 332, 163 (arXiv:astro-ph/0409677)

Zhang C.M., 1998, A\&A, 330, 195 